\title{
Modification and ab-initio spectroscopic application of modified commerce terahertz spectrometer by using homemade parts
}

\author{
D.M. Lubenko ${ }^{1,2}$, V.F. Losev ${ }^{1,2,3}$, Yu.M. Andreev ${ }^{2,4}$, K.A. Kokh²,5, G.V. Lanskii ${ }^{2,4}$, V.A. \\ Svetlichnyi ${ }^{2}$ \\ ${ }^{1}$ High Current Electronics Institute SB RAS, 2/3, Akademicheskii Ave., 634055, Tomsk, Russia, \\ lideru@gmail.com; \\ ${ }^{2}$ National Research Tomsk State University, 36, Lenina Ave., 634050, Tomsk, Russia; \\ ${ }^{3}$ National Research Tomsk Polytechnic University, 30 Lenina Ave., 634050, Tomsk, Russia; \\ ${ }^{4}$ Institute of Monitoring of Climatic and Ecological Systems SB RAS, 10/3 Akademicheskii Ave., \\ 634055, Tomsk, Russia; \\ ${ }^{5}$ Institute of Geology and Mineralogy SB RAS, 3, Koptyug Str., 630090, Novosibirsk, Russia.
}

\begin{abstract}
Ab-initio study on modification of commerce terahertz spectrometer with time resolution Z-3 (Zomega, USA) by substitution of ZnTe \& GaP detectors and LT-GaAs generator for homemade of pure and S-doped GaSe is carried out. It was established that in spite of not optimized parameters pure and doped GaSe: $\mathrm{S}(0.3$ mass\%) crystal are comparable, relatively, in generation efficiency and detection sensitivity to commerce units due to lower nonlinear optical loss and much higher damage threshold. The advantages are in force from pump fluences of below $5 \mathrm{~mJ} / \mathrm{cm}^{2}$ for pure GaSe. The closer S-doping to optimal concentration, the lover fluences resulting in the advantages. Pure and S-doped GaSe demonstrate higher reliability and larger dynamic range of operation. Recorded absorption spectra well match known spectra.
\end{abstract}

Keywords: GaSe crystal, doped crystals, nonlinear optics, $\mathrm{THz}$

\section{INTRODUCTION}

The last three decades have witnessed an unprecedented advance in the terahertz (THz) science and technology. It includes advance in design of uncooled sensitive detectors and effective generators of $\mathrm{THz}$ emissions. Traditionally used, huge and expensive terahertz instruments have evolved into commercially available top-table systems. On current time, terahertz time domain spectrometers (THz-TDS) are in wide use in applied spectroscopy in lab conditions. Anyway, further improvement of their key parts to extend operation range and improve reliability until possibility of out-of-door use are still desirable, as well as decrease of price.

Isotropic semiconductor ZnTe is amongst the most popular material for free space (also referred to as electrooptical) detectors and optical rectifiers (also referred to as $\mathrm{THz}$ generators). It is due to a large electrooptical coefficient that is linearly related with the second order nonlinear susceptibility coefficient $\mathrm{d}_{14}=90 \mathrm{pm} / \mathrm{V}$ (at $10.6 \mu \mathrm{m}$ ) and good mechanical properties. For efficient operation as a THz rectifier, ZnTe should be pumped by $760-840 \mathrm{~nm}$ fs lasers that is lucky well in coincidence with the operation range $680-1080 \mathrm{~nm}$ of available highly efficient $\mathrm{fs}$ Ti:Sapphire laser. However, the coherence length of $\mathrm{ZnTe}$ limits its suitable thickness and finally rectification efficiency. Achieved rectification efficiency is additionally limited by the large, $4 \mathrm{~cm} / \mathrm{GW}$, two-photon absorption coefficient at near IR wavelengths and large, $>40 \mathrm{~cm}^{-1}$, linear absorption coefficient at frequencies over $3.5 \mathrm{THz}$. In spite of the almost two times lower electroopitical/nonlinear coefficient, isotropic semiconductor GaP demonstrates higher rectification efficiency into high frequency range over $3.5 \mathrm{THz}$ to that of $\mathrm{ZnTe}$ due to lower optical losses. Potentials of other cubic semiconductors, such as GaAs, LT-GaAs, InP, InAs, etc. as THz detectors and generators are close to the above-mentioned crystals and add 
each other in different characteristics only in some details. Anisotropic organic DAST crystal is characterized by principally larger electrooptical and nonlinear coefficients, as well as by much lower absorption coefficient in comparison with those for semiconductor crystals. Its large anisotropy allows phase matched, i.e. much more efficient, rectification into $\mathrm{THz}$ range that is also referred to as down-conversion. Unfortunately, DAST is hydroscopic and possesses a low damage threshold. Besides, it is hard to grow as centimeter size samples. So such, sub-micrometer thick films of this material are dominantly used as optical rectifiers of low intensity beams ${ }^{1,2}$.

The last two decades, birefringent GaSe crystal was amongst the most used anisotropic crystals for not phase matched (optical rectification) and phase matched (down-conversion) into $\mathrm{THz}$ range of near IR emission in lab conditions ${ }^{1}$. It is due to extra ordinary physical properties: the extreme wide transparency range $0.62-20 \mu \mathrm{m}$ continue from 50-60 $\mu \mathrm{m}$ further into the $\mathrm{THz}$ range, low dispersion, large electrooptical coefficient $\mathrm{r}_{41}=14.4 \mathrm{pm} / \mathrm{V}$ and second order susceptibility coefficient $\mathrm{d}_{22}=24.3 \mathrm{pm} / \mathrm{V}$ in the $\mathrm{THz}$ range ${ }^{3}$. The giant birefringence $\mathrm{B}=0.35$ in the mid-IR ${ }^{4}$ and about 0.79 in the $\mathrm{THz}$ range $^{5}$ that allows phase-matched down-conversion of long and ultrashort pulses almost all over the entire transparency range ${ }^{3}$. It possesses low nonlinear absorption coefficient. GaSe is cheap in synthesis and can be manufactured as centimeter size samples. ${ }^{6}$ Unfortunately, poor mechanical properties (measured hardness is almost " 0 " in Mohs scale) and relatively low optical quality (absorption coefficient $\geq 0.1-0.2 \mathrm{~cm}^{-1}$ at main transparency window and over $0.5 \mathrm{~cm}^{-1}$ at frequencies over $1 \mathrm{THz}$ ) caused by the layered structure and technologically uncontrolled point and micro defects have so far kept back commerce applications.

In order to fully exploit the potential of GaSe and to expand its applications, it is necessary to overcome these limitations. Fortunately, GaSe is an excellent matrix for doping with different elements. Appropriate doping by various isovalent elements that form isostructural compounds, e.g. sulphur (S), tellurium (Te), indium (In), etc., is an effective way to obtain improvements of GaSe physical properties ${ }^{7,8}$. The optimal Te-doping of 0.38 mass. \% noticeably decreases the number of point defects and stacking faults and increases damage threshold for from 15 to $20 \%$ that is resulting in up to 2 times improved phase matched frequency conversion efficiency within the mid-IR. Rectification efficiency of pure and Te-doped GaSe into the THz range evidently prevails that in $\mathrm{ZnTe}$ at pump fluence of $>4.5 \mathrm{~mJ} / \mathrm{cm}^{2}$ due to higher damage threshold caused by lower nonlinear absorption coefficient ${ }^{9,10,11}$. The optimal S-doping is leading to the most impressive results in mid-IR efficiency (up to 3 times at fixed pulse intensity; $50 \%$ increased hardness) ${ }^{7,12}$. The efficiency of phase-matched down-conversion of long (ns) pulses into the THz range is also improving for 3 times ${ }^{13-16}$. Recently, it was established that optimal S-doping also increases the damage threshold for from 4 to 5 times ${ }^{17,18}$. At the reasonably high pump fluence, the phase-matched frequency conversion efficiency within the mid-IR raises up to 15 times $^{19-21}$. Impressive results were demonstrated on frequency conversion in S-doped GaSe of ultrashort ( $\mathrm{ps}$ and $\mathrm{fs}$ ) pulses into mid-IR ${ }^{13,22}$. However, very limited data are available yet on efficiency of not phase matched and phase matched optical rectification of fs pulses in S-doped GaSe crystals into the $\mathrm{THz}$ range ${ }^{23}$. No attempts were made on application of pure and doped GaSe crystals in commerce devises as $\mathrm{THz}$ detector or generator.

In this work, we tried our best for the first time to our knowledge to use S-doped GaSe crystals as generators and EO detectors in commerce THz-TDS Z-3 (Zomega, USA). Exfoliated samples from the as-grown boulles were used. No additional treatment or attempts on optimization of crystal length or modification of the spectrometer optical set up were made.

\section{CRYSTAL GROWTH}

For this study, pure and doped GaSe crystals were obtained by modified two-zone ${ }^{24}$ syntheses method. In accordance with this method, Ga is placed in the "hot" zone of the ampoule, while the other part with Se is slowly heated in the "cold" zone according to the progress of synthesis reaction. The starting materials for the synthesis were Ga 99.9997, Se 99,99 and S 99.95, which were additionally purified by remelting in a continuously evacuated ampoule. We preferred to use single zone furnace with gradual moving of the ampoule inside ${ }^{25}$. This technique seems to be very reliable, because one may estimate the vapor pressure inside the ampoule visually by its color. To improve optical quality of the synthesized compound synthesis is conducting by using quartz ampoules charged with a large amount, up to $65 \%$ in volume, to decrease rest gases quantity and as a result to improve the material quality. Growth ampoules covered with pyrolytic carbon are used to exclude interaction with the quartz wall to prevent deformation of grown boulles. Growth oven with heat field rotation was used to make melt uniform and crystallization front thinner and finally grown crystals of high optical quality. Other details on the growth process used can be find elsewhere ${ }^{25,26}$. Visual inspection of as-grown crystals didn't show any color differences between initial and final sections of boules. Its high optical quality can be 
estimated by naked eye, evident in its transparency and homogenity. Eutectic was negligible and layered GaSe structure could be seen through it. So, end section of the boule also could be easy cleaved that confirm its top quality. Samples made were made simple exfoliation by using blade. Any additional processing was not exploited.

\section{CRYSTAL CHARACTERIZATION}

The physical properties of the S-doped GaSe were characterized at room temperature. Scanning electron microscopy (SEM) with a SEM Quanta 200 3D (FEI, Netherlands) microscope was used in the study of surface morphology, and Ga and Se contents after providing with EDAX ECON VI micro analyzer. An X-ray diffractometer Shimadzu XRD 6000 (Japan), and a transmission electron microscope (TEM) CM12 (Philips, Netherlands) for measurement used in the SAED method were also implode in analyzing structure. UV-visible-near-IR transmission spectra were recorded by a Cary 100 Scan (Varian Inc.) spectrophotometer over the spectral range $190-900 \mathrm{~nm}$ with a spectral resolution $\Delta \lambda 0.2-4 \mathrm{~nm} \mathrm{~nm}$. The measurements of linear optical properties at $0.2-4 \mathrm{THz}$ were performed at room temperature using a THz-TDS described elsewhere ${ }^{27}$.

Only Ga, Se and S peaks were obsearved in the EDAX spectra. The diffraction patterns clearly confirm the high quality of the crystalline structure. We found that in unpolarised light the absorption coefficient $\alpha$ for GaSe crystals does not exceed $0.05 \mathrm{~cm}^{-1}$ within their maximal transparency range that is 2-3 times lower to that for GaSe crystals grown by conventional vertical Bridgman technology. Optical quality of S-doped crystals is found depending on S-content. At identified optimal doping concentration of $2-3$ mass $\%$ of $S$ they demonstrated once more again decrease in the absorption coefficient for 2-3 times at both mid-IR and THz frequencies. Short-wave edge of doped crystals linear shifts toward short waves proportional to the S-content down to $0.54 \mu \mathrm{m}$ at 11 mass $\% \mathrm{~S}$ that significantly decrease nonlinear two-photon absorption coefficient. Besides, optical quality of exfoliated samples became evidently of higher quality due to decreased cleavage. Improved linear and nonlinear optical properties of S-doped GaSe crystals lead to 4-5 times larger damage threshold that allows increasing frequency conversion efficiency by increasing pump intensity.

\section{EXPERIMENTAL}

The terahertz time-domain spectrometer Z-3 (Zomega, USA) used a standard configuration incorporating a femtosecond laser, four off-axis parabolic mirrors, a biased LT-GaAs emitter, and electro-optic detector with changeable ZnTe and GaP crystals, and balanced Si photodiodes. The frequency resolution was 5-200 GHz. Pump laser system based on Ti:Sapphire laser with external resonator and has next parameters: $950 \mathrm{~nm}, 50 \mathrm{fs}, 150 \mathrm{~mW}$. Master Ti:Sapphire laser system was also of classic type. It should be noted that parameters of Z-3 THz-TDS are not extra for spectroscopic applications but anyway, it is very useful for our study. Older design THz-TDS Z-3 makes simple substitution of its key units, such as emitter or detector, by homemade ones. External view at the Z-3 spectrometer is shown in Fig.1.

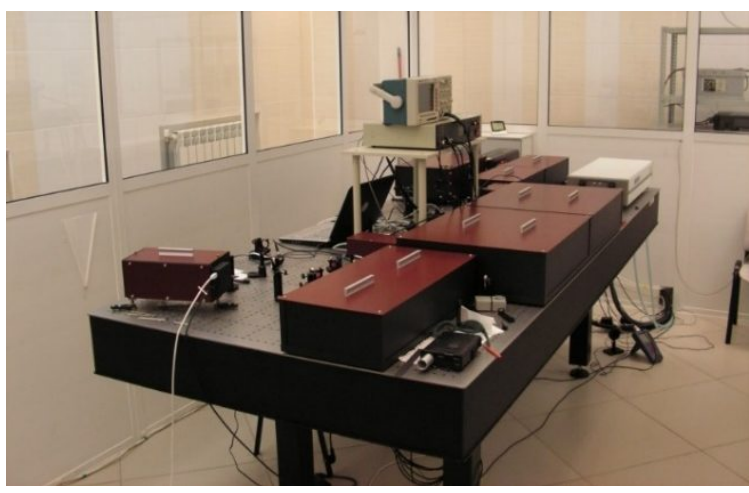

a

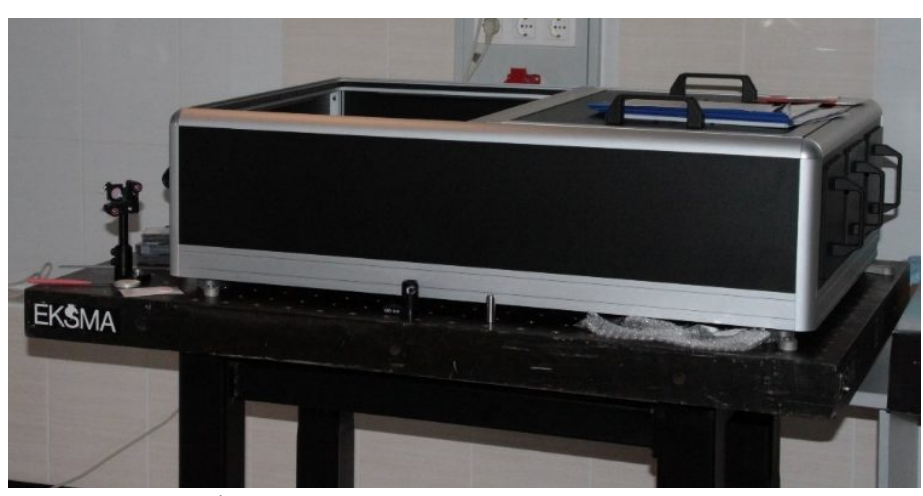

b 

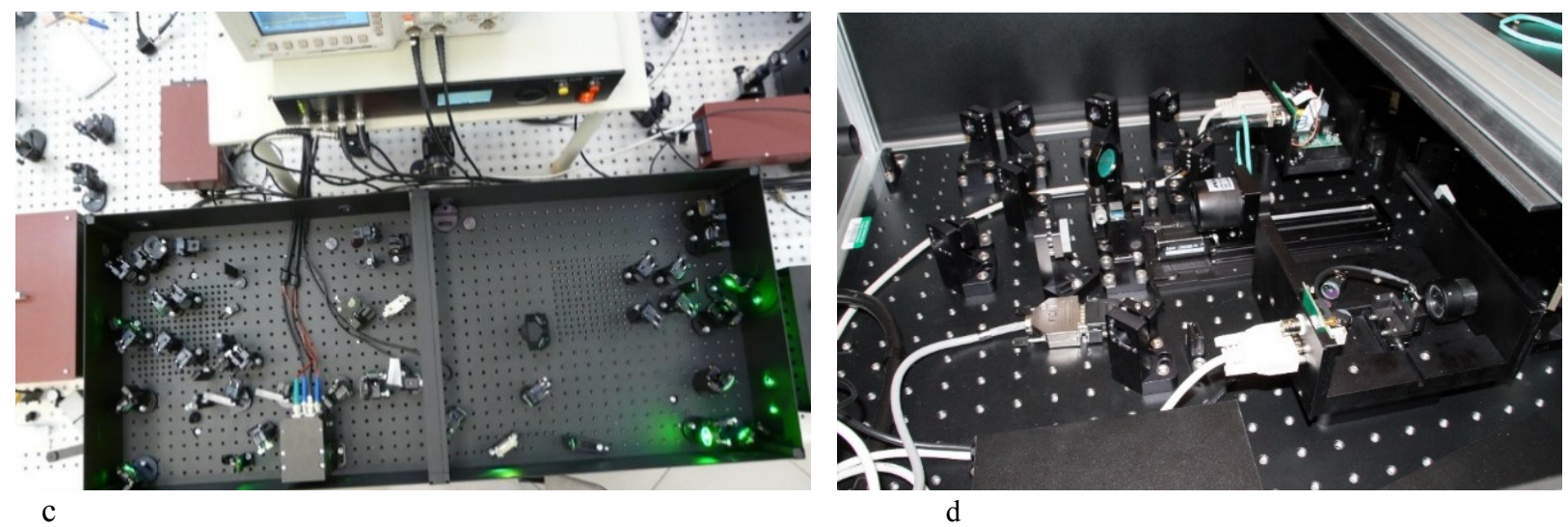

Fig. 1. (a) External and (b) inside view at Z-3 THz-TDS, (c) pump fs laser system and (d) inside view at master laser.

Output parameters of the pump system can be scaled up. External view on substituted and installed crystals are shown in Fig. 2.

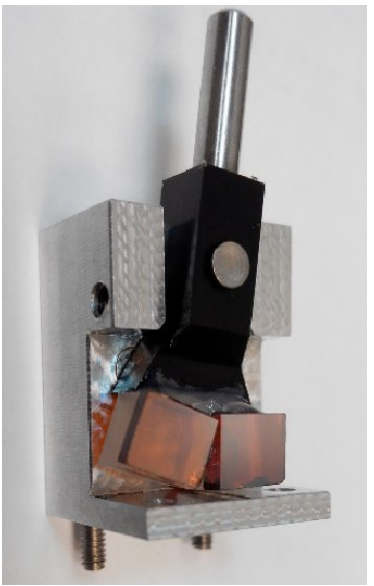

a

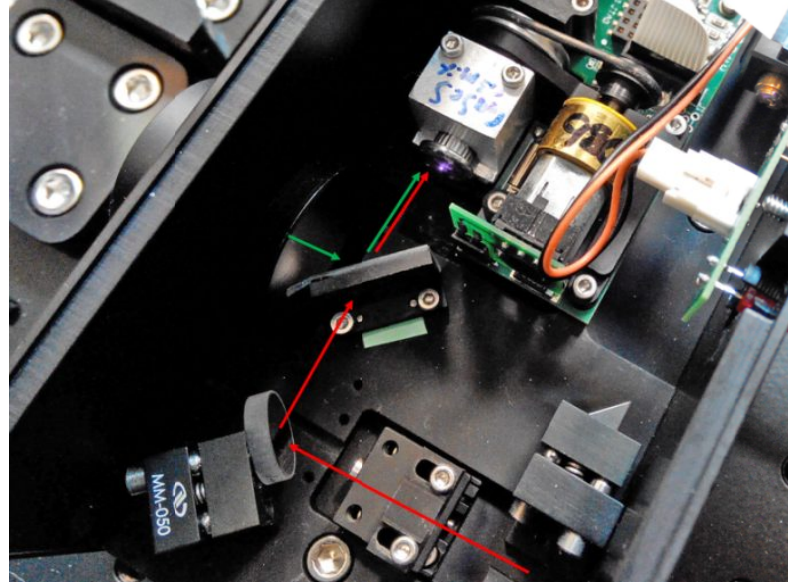

b

Fig. 2. Crystals used in THz-TDS Z-3: (a) ZnTe (left) and GaP (right), (b) GaSe:S in the use

Pure and doped GaSe crystals were mainly 2-2.5 times thinner.

\section{RESULT AND DISCUSSION}

Absorption spectra recorded by the commerce THz-TDS Z-3 are shown in Fig.2. Absorption peak of the $\mathrm{E}^{(2)}$ rigid phonon mode in $\mathrm{GaSe}$ at $0.59 \mathrm{THz}$ presented in Fig. $2 \mathrm{~b}$ well meets known data ${ }^{20}$. Its large intensity and narrow spectral bandwidth yet once again confirms high optical quality of grown GaSe crystal ${ }^{9}$. Limited SNR does not allow observation of the smooth absorption structure at frequencies over 1.5-2 THz.

First, we studied possibility of application of pure and S-doped GaSe crystals as the free space detector. Substitution of the basic ZnTe and GaP detectors by $0.6 \mathrm{~mm} \mathrm{GaSe}$ or $0.8 \mathrm{~mm}$ GaSe:S (0.3 mass\%) was made one by one. It allowed us to record successfully THz emission from LT-GaAs dipole antenna of THz-TDS Z-3 (Fig.3). No optimization of crystal thickness were made but in Fig. 3 it is evidently seen that positive result of the substitution was achieved in spite of the smaller thickness and not maximized intensity of the reference Ti:Sapphire laser beam. Even not optimally, 0.3 mass $\%$, S-doped GaSe demonstrated 30\% higher sensitivity than that of GaSe detector. It is due to improved optical quality and lattice structure. GaSe:S (0.3 mass\%) crystal with not optimized length demonstrates higher sensitivity even than commerce GaP detector. It $\mathrm{t}$ seems that pure GaSe has at least close sensitivity. It is because lower nonlinear absorption 
coefficient). It and improved optical quality of grown crystals is leading to higher damage threshold ${ }^{17,18}$. So, intensity of the reference beam and finally sensitivity can be further improved for GaSe and S-doped GaSe detectors by increasing pump intensity.

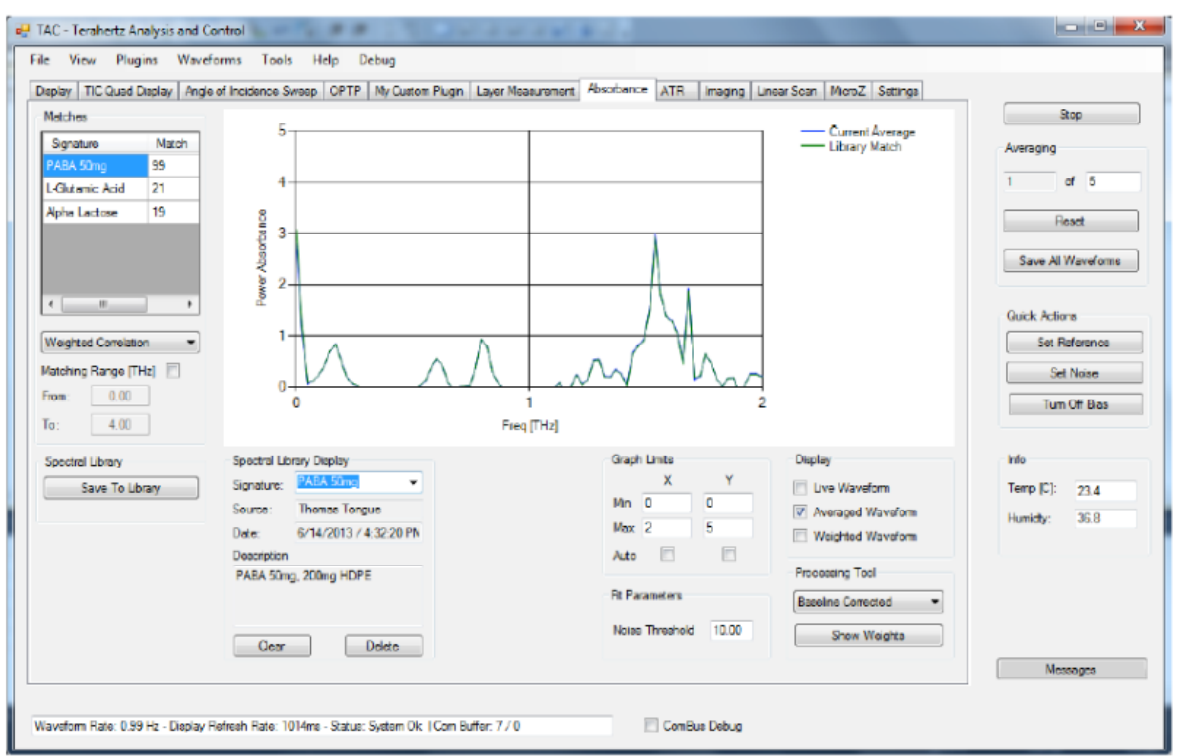

a

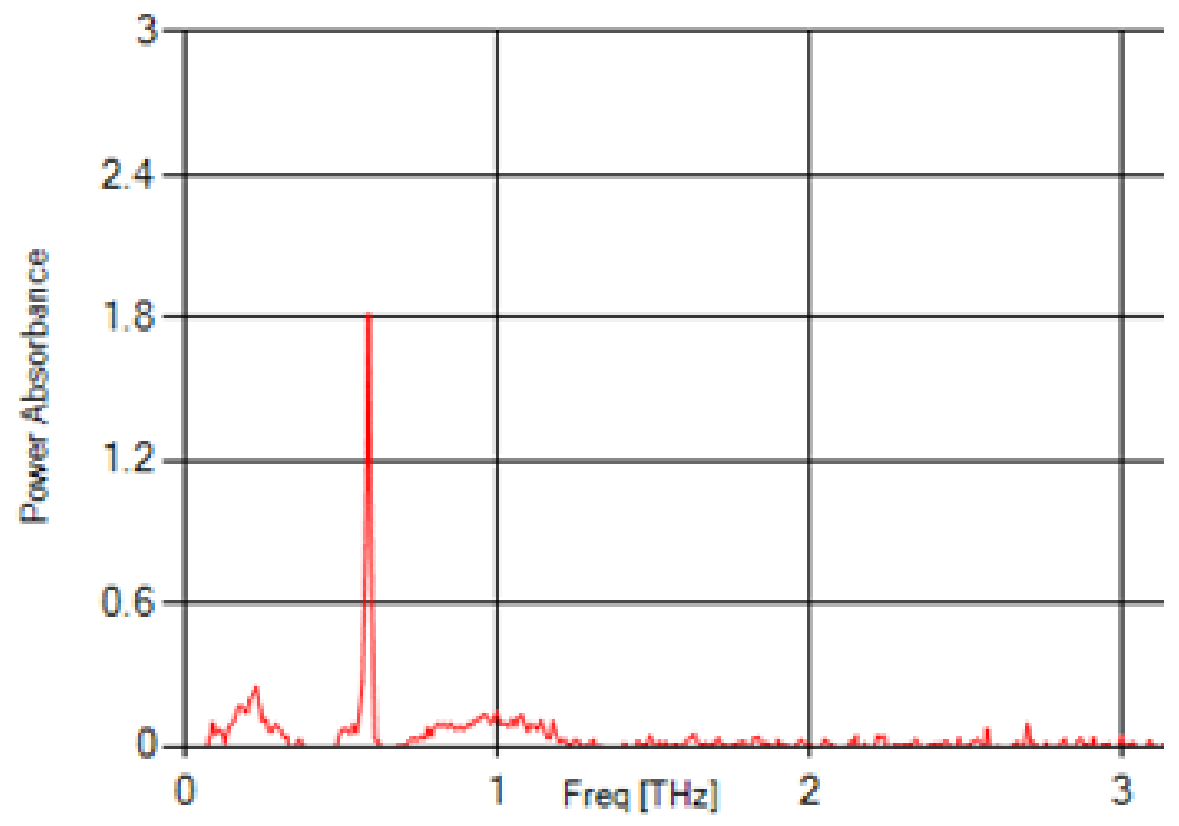

b

Fig.2. An example of absorption spectrum of (a) a drug tablet as-given in the manual and (b) absorption spectrum of GaSe with absorption peak of the rigid phonon mode $\mathrm{E}^{\prime(2)}$ at $0.59 \mathrm{THz}$ recorded by the THz-TDS Z-3 in lab conditions. 


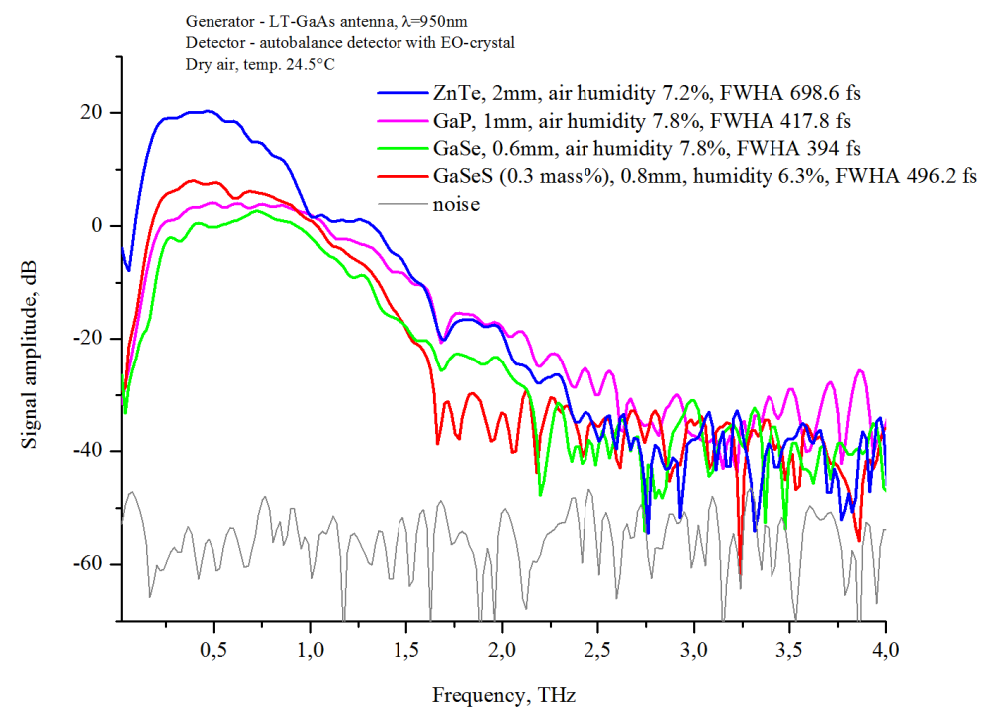

Fig.3. THz pulse spectra on the output of LT-GaAs dipole antenna recorded by different detectors at identical experimental conditions.

Rectification (THz generation) efficiency is also depends on the pump intensity that is higher for pure and doped GaSe crystals. Thus, substitution of the ZnTe detector for pure GaSe and doped GaSe crystals should resulted in higher generation efficiency. It was observed beginning from fixed pump fluences depending on the doping concentration (Fig.4).

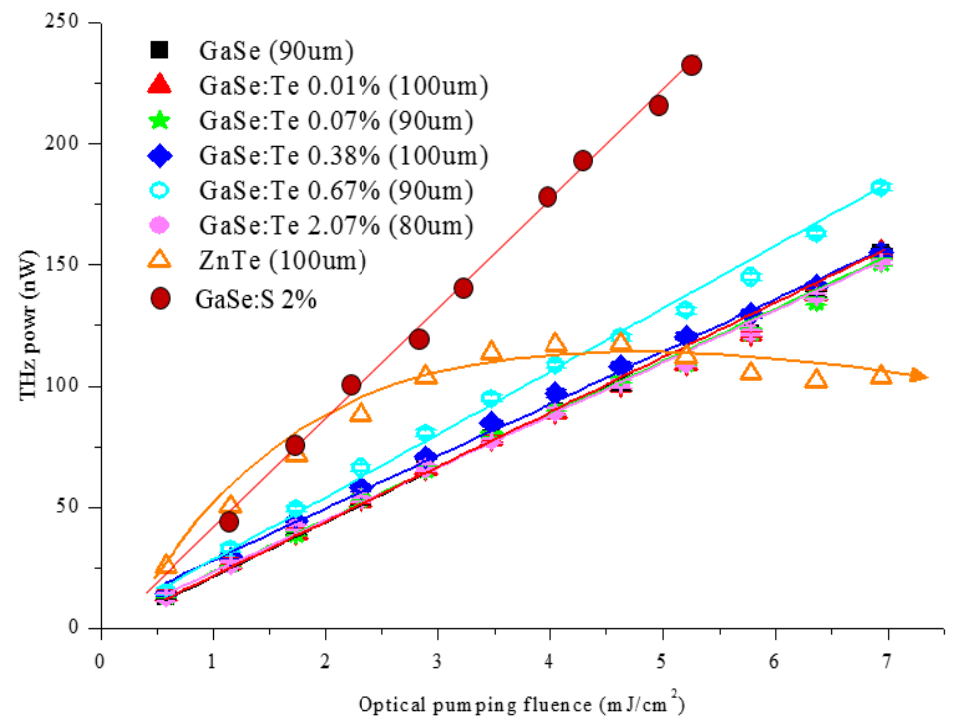

Fig.4. THz emission power on the output of rectifiers versus pump fluence.

In Fig. 4 it is seen that GaSe is characterized by higher efficiency in comparison with that for ZnTe under pump fluences over $5 \mathrm{~mJ} / \mathrm{cm}^{2}$. Advantage for optimally 2 mass\% S-doped GaSe crystal appears from fluences of about $2 \mathrm{~mJ} / \mathrm{cm}^{2}$. Moreover, in difference to cubic GaP and ZnTe, efficiency of pure and doped GaSe crystals can be increased significantly by phase matched optical rectification (down-conversion) that is a subject of the future study. Besides, Sdoped GaSe allows someone to realize unprecedented broadband generation and detection of $\mathrm{THz}$ emission. It is also important for practice that pure and doped GaSe crystals are simple and cheap in syntheses and sample fabrication for example. Their high optical damage threshold resulted in high reliability. Moreover, as it is seen in Fig. 4, pure and 
doped GaSe crystals are characterizing by much larger dynamic range as generators, as well as detectors due to a range of their physical properties. So such, further careful study of pure and doped GaSe crystal applicability as optical rectifies and detectors seems to be reasonable. It is necessary to outline that current study are just ab-initio study in this field.

Some results of ab-initio spectroscopic study of crystals and drugs are shown in the following Fig. 5-7

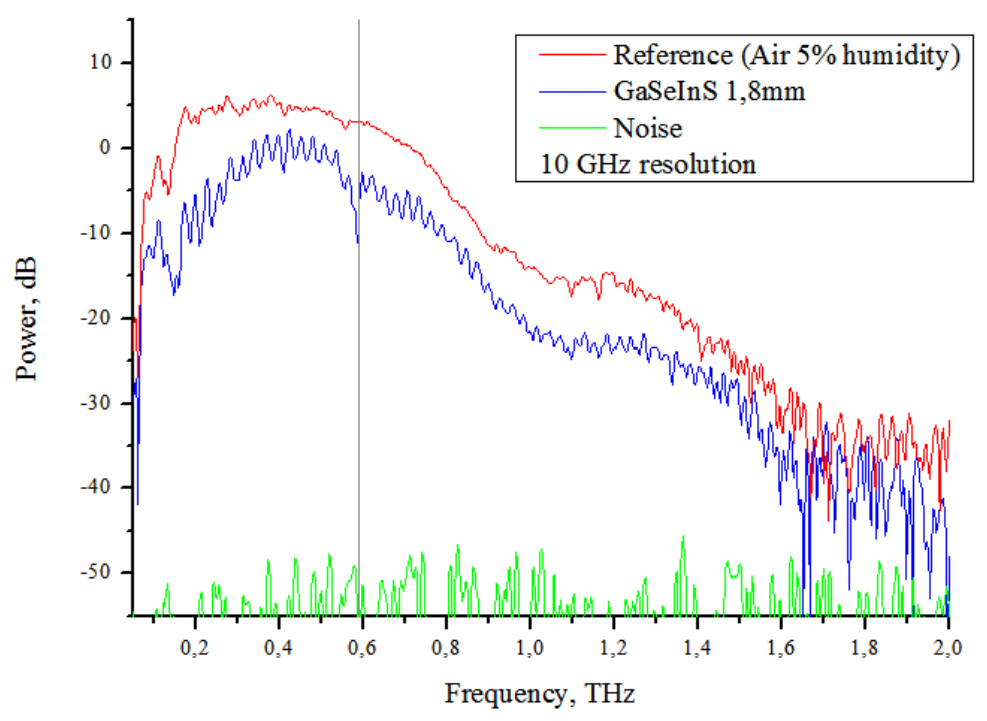

Fig. 5. Absorption spectra of GaSe:InSe(1 mass\%) in the THz range recorded with spectral resolution $10 \mathrm{GHz}$.

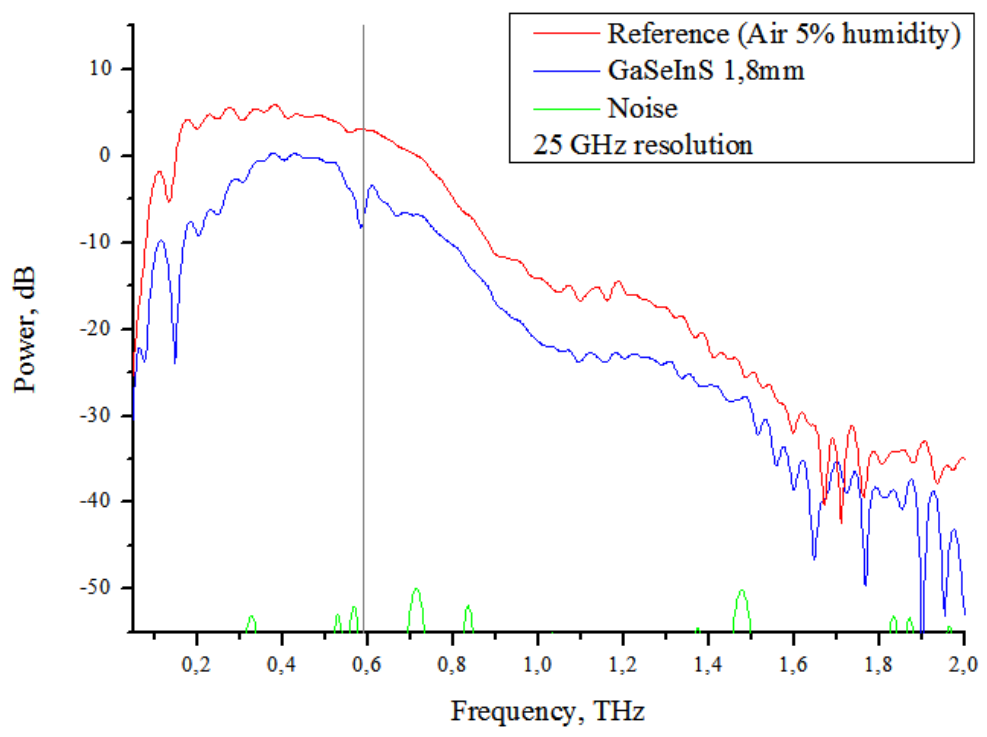

Fig. 6. Absorption spectra of GaSe:InSe(1 mass\%) in the THz range recorded with spectral resolution $25 \mathrm{GHz}$. 


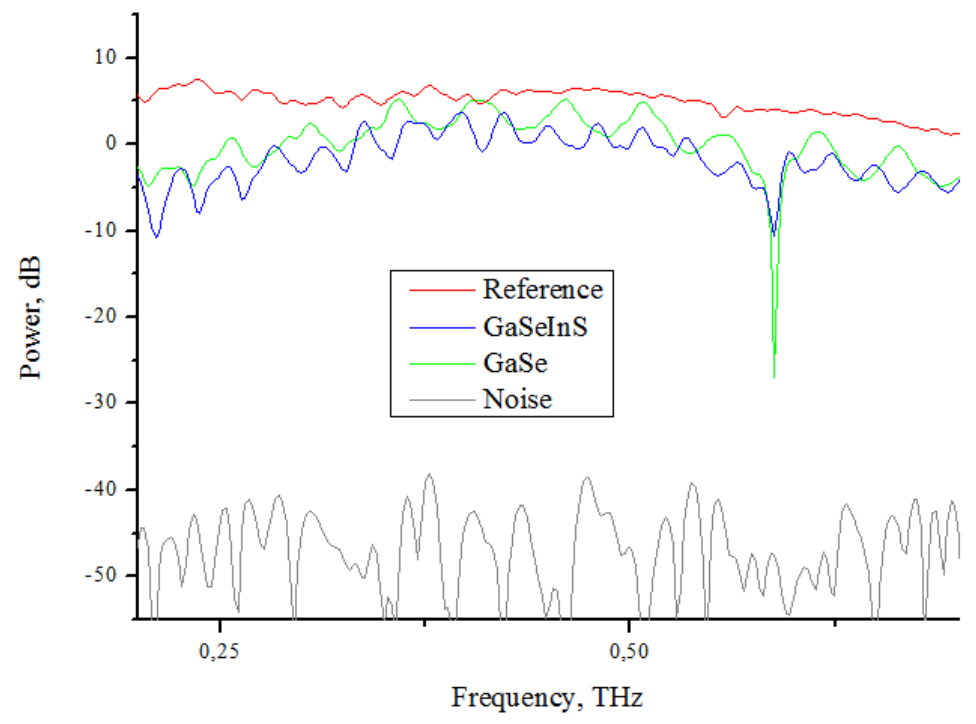

Fig.7. Plots of THz absorption spectra of pure GaSe and GaSe:InSe(1 mass\%) crystals with spectral resolution $10 \mathrm{GHz}$.

In Fig. 5 - 7 it is seen that recorded spectra are of the same quality as exampled in the Z-3 manual (Fig. 2a). Besides, it is seen that absorption peak of the rigid phonon mode in GaSe at $0.59 \mathrm{THz}$ is decreasing with the increasing of the spectral resolution and doping with InS (It is in fact four component $\mathrm{Ga}_{1-\mathrm{x}} \mathrm{In}_{\mathrm{x}} \mathrm{Se}_{1-\mathrm{y}} \mathrm{S}_{\mathrm{y}}$ crystal) in full accordance with known data ${ }^{20}$. Recorded absorption spectra for aspirin shown in Fig. 8. In Fig. 8 it is seen that one run (no averaging) THz absorption spectrum of aspirin well match absorption spectrum from the data bank. It is the common case in the study carried out.

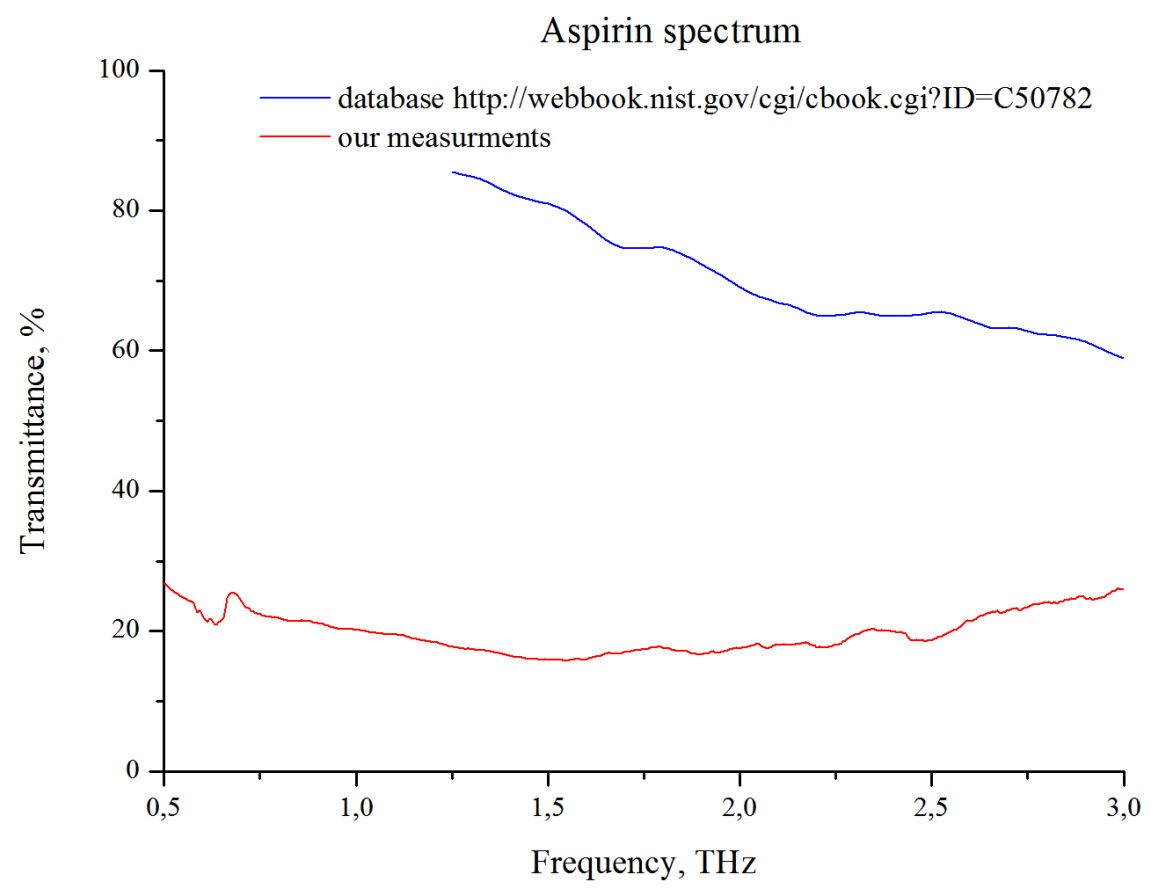

Fig.8. Transmission spectra of aspirin as presented in internet data base (red line) and recorded with modified Z-3 THz-TDS. 


\section{CONCLUSION}

Ab-initio study on modification of commerce terahertz spectrometer with time resolution Z-3 (Zomega, USA) by substitution of ZnTe \& GaP detectors and LT-GaAs generator for homemade of pure and S-doped GaSe is carried out. It was established that in spite of not optimized parameters pure and doped GaSe:S (0.3 mass\%) crystal are comparable, relatively, in generation efficiency and detection sensitivity. It was established that it is due to lower nonlinear optical loss and much higher damage threshold. The advantages are in force from pump fluences of below $5 \mathrm{~mJ} / \mathrm{cm}^{2}$ for pure GaSe. The closer S-doping to optimal concentration, the lover fluences resulting in the advantages. Pure and S-doped GaSe demonstrate higher reliability and larger dynamic range of operation. Recorded absorption spectra well match known spectra.

Acknowledgment: The experimental study was supported by the Russian Scientific Fund (Project No. 15-19-10021), the growth crystals and its study was supported by Russian Ministry of Education and Science (14.594.21.0001, RFMEFI59414X0001) and the Tomsk State University (8.1.51.2015).

\section{REFERENCES}

[1] Lee Y.-S., "Principle of terahertz science and technology," Springer, New York, 340 (2008).

[2] Martienssen W., Warlimont H., "Handbook of condensed matter and materials data," Springer-Verlag, Berlin, 1120 (2005).

[3] Tochitsky S.Ya., Sung Ch., Trubnick S.E., Joshi Ch., Vodopyanov K.L., "High-power tunable, 0.5-3 THz radiation source based on nonlinear difference frequency mixing of $\mathrm{CO} 2$ laser lines," J. Opt. Soc. Am. B. V. 24., N. 9, 2509-2516 (2007).

[4] Nikogosyan D.N., "Nonlinear optical crystals: a complete survey" Springer, 427 (2005).

[5] Nazarov M.M., Shkurinov A.P., Angeluts A.A., Sapozhnikov D.A., "On the choice of nonlinear optical and semiconductor converters of femtosecond laser pulses into terahertz range" Radiophysics and Quantum Electronics, V. 52, No. 8, 536-545 (2009).

[6] Kokh K.A., Andreev Yu.M., Svetlichnyi V.A., Lanskii G.V., Kokh A.E., "Growth of GaSe and GaS single crystals," Cryst. Res. Technol. V. 46, No. 4, 327-330 (2011).

[7] Zhang H.-Z., Kang Z.-H., Jiang Yu., Gao J.-Yu., Wu F.-G., Feng Z.-S., Andreev Yu.M., Lanskii G.V., Morozov A.N., Sachkova E.I., Sarkisov S.Yu., "SHG phase matching in GaSe and mixed $\mathrm{GaSe}_{1-\mathrm{x}} \mathrm{S}_{\mathrm{x}}, \mathrm{x} \leq 0.412$, crystals at room temperature," Optics Express, V. 16, N. 13, 9951-9957 (2008).

[8] Feng Z.-S., Kang Z.-H., Wu F.-G., Gao J.-Yu., Jiang Yu., Zhang H.-Z., Andreev Yu.M., Lanskii G.V., Atuchin V.V., Gavrilova T.A., "SHG in doped GaSe:In crystals," Optics Express V. 16, N. 13, 9978-9985 (2008).

[9] Andreev Y.M., Lanskii G.V., Shaiduko A.V., Vaitulevich E.A., Svetlichnyi V.A., Losev V.F., Kokh K.A., Lubenko D.M., Soldatov A.N., "Optimal doping of GaSe for nonlinear optical applications," Russian Physics Journal V.56, No.11, 1250-125 (2014).

[10] Ku S.-A., Chu W.-C., Luo C.-W., Andreev Yu.M., Lanskii G.V., Shaiduko A.V., Izaak T.I., Svetlichnyi V.A., "Optimal Te-doping in GaSe for non-linear applications," Optics Express, V.20, No. 5, 5029-5037 (2012).

[11] Chu W.-C., Ku S.-A., Wang H. J., Luo C.-W., Andreev Yu. M., Lanskii G.V., Kobayashi T., "Widely linear and non-phase-matched optics-to-THz conversion on GaSe:Te crystals," Optics Letters, V.37, No.5, 945-947 (2012).

[12] Guo J., Kang Z.-H., Feng Z.-S., Jiang Y., Gao J.-Y., Xie J.-J., Zhang L.-M., Atuchin V. V., Andreev Yu.M. , Lanskii G.V., Shaiduko A.V., "Tellurium and sulphur doped GaSe for mid-IR applications," Applied Physics B: Lasers and Optics, V. 108, No. 3, 545-552 (2012).

[13] Petrov V., Panyutin V.L., Tyazhev A., Marchev G., Zagumennyi AI., et al., " $\mathrm{GaS}_{0.4} \mathrm{Se}_{0.6}$ : Relevant properties and potential for $1064 \mathrm{~nm}$ pumped mid-IR OPOs and OPGs operating above $5 \mu \mathrm{m}$," Laser Phys., V. 21, 774781 (2011).

[14] Miyata K., Marchev G., Tyazhev A., Panyutin V., Petrov V., "Picosecond mid-infrared optical parametric amplifier based on the wide-bandgap $\mathrm{GaS}_{0.4} \mathrm{Se}_{0.6}$ pumped by a Nd:YAG laser system at $1064 \mathrm{~nm}$," Opt. Lett., V. $36,1785-1787$ (2011). 
[15] Huang J., Huang Zh., Tong J., Ouyang Ch., Chu J., Andreev Yu.M., Kokh K.A., Lanskii G.V., Shaiduko A.V., "Intensive terahertz emission from $\mathrm{GaSe}_{0.91} \mathrm{~S}_{0.09}$ under collinear difference frequency generation," Appl. Phys. Lett., V. 103, 81104 (2013).

[16] Xie J.-J., Guo J., Zhang L.-M., Chen F., Jiang K., Andreev Yu.M., Atuchin V.V., Gorobets V.A., Lanskii G.V., Svetlichnyi V.A., Shaiduko A.V., "Frequency conversion of nanosecond $\mathrm{CO}_{2}$-laser into $\mathrm{THz}$ range in doped GaSe crystal," Basic Problems of Material Science (BPMS), V. 9, No. 4, 486-494 (2012).

[17] Guo J., Li D.-J., Xie J.-J., Zhang L.-M., Feng Z.-S., Andreev Yu.M., Kokh K.A., Lanskii G.V., Potekaev A.I., Shaiduko A.V., Svetlichnyi V.A., "Limit pump intensity for sulfur-doped gallium selenide crystals," Laser Phys. Lett., V.11, No.5, 055401 (2014).

[18] Feng Z.-S., Kang Z.-H., Li X.-M., Gao J.-Y., Andreev Yu. M., Atuchin V.V., Kokh K. A., Lanskii G. V., Potekaev A.I., Shaiduko A.V., Svetlichnyi V.A., "Impact of fs and ns pulses on solid solution crystals $\mathrm{Ga}_{1-}$ ${ }_{x} \operatorname{In}_{\mathrm{x}} \mathrm{Se}$ and $\mathrm{GaSe}_{1-\mathrm{x}} \mathrm{S}_{\mathrm{x}}$," AIP Advances, Vol.4, Issue 3, 037104(1-6) (2014).

[19] Kokh K.A., Molloy J.F., Naftaly M., Andreev YuM., Svetlichnyi V.A., Lanskii G.V., Lapin I.N., Izaak T.I., Kokh A.E., "Growth and optical properties of solid solution crystals $\mathrm{GaSe}_{1-\mathrm{x}} \mathrm{S}_{\mathrm{x}}$," Materials Chemistry and Physics, 154, 152-157 (2015).

[20] Molloy J.F., Naftaly M., Andreev Yu.M., Kokh K.A., Lanskii G.V., Svetlichnyi V.A., “Absorption anisotropy in sulfur doped gallium selenide crystals studied by THz-TDS," Optical Materials Express, Vol. 4, Issue 11, 2451-2459 (2014).

[21] Guo J., Xie J.J., Li D.J., Yang G.L., Chen F., Wang C.R., Zhang L.M., Andreev Yu.M., Kokh K.A., Lanskii G.V., Svetlichnyi. V.A., "Doped GaSe crystals for laser frequency conversion," Light: Science \& Applications, V. 4, e362 (2015). DOI: 10.1038/1sa.2015.135.

[22] Marchev G., Tyazhev A., Panyutin V., Petrov V., Noack F., Miyata K., Griepentrog M., "Some properties of the mixed GaS0.4Se0.6 nonlinear crystal in comparison to GaSe," Proceedings of SPIE V.7917, 79171G (2011).

[23] Ku S.A., Luo C.W., Andreev Yu.M., Lanskii G.V., „Comment on “ $\mathrm{GaSe}_{1-\mathrm{x}} \mathrm{S}_{\mathrm{x}}$ and $\mathrm{GaSe}_{1-\mathrm{x}} \mathrm{Te}_{\mathrm{x}}$ thick crystals for broadband terahertz pulses generation” [Applied Physics Letters 99, 081105 (2011)],” Appl. Phys. Lett. 100: 136103 (2012).

[24] Vohl P., "Synthesis and crystal growth of $\mathrm{CdGeP}_{2}$," J. Electron Mater, V.8, N4, 517-522 (1979).

[25] Kokh K.A., Andreev Yu.M., Svetlichnyi V.A., Lanskii G.V., Kokh A.E., "Growth of GaSe and GaS single crystals," Cryst. Res. Technol, V.46, 327-330 (2011).

[26] Kokh K.A., Nenashev B.G., Kokh A.E., Shvedenkov G.Yu., "Application of a rotating heat field in BridgmanStockbarger crystal growth,” J. Cryst. Growth, V. 275, No. 1-2, e2129-e2134 (2005).

[27] Molloy J. F., Naftaly M., Andreev Yu. M., Lanskii G. V., Lapin I. N., Potekaev A. I., Kokh K. A., Shabalina A. V., Shaiduko A. V., Svetlichnyi V. A., "Dispersion properties of GaS studied by THz-TDS," CrystEngComm $16,1995-2000$ (2014).

[28]Allakhverdiev K.R., Guliev R.I., Salaev E.Yu., Smirnov V.V., "Investigation of linear and nonlinear optical properties of $\mathrm{GaSe}_{\mathrm{x}} \mathrm{Se}_{1-\mathrm{x}}$ crystals," Soviet J. Quantum Electron., V.12, No.7, 947-948 (1982). 